\title{
Desparasitação intestinal sistemática em idade pediátrica: uma revisão baseada na evidência
}

Vanessa Xavier,* Brenda Domingues, ${ }^{*}$ Teresa Marcos**

Objectivos: Determinar se a desparasitação intestinal sistemática com anti-helmínticos, em idade pediátrica, influencia o desenvolvimento estaturo-ponderal, cognitivo, o aproveitamento escolar e a morbimortalidade.

Fontes de Dados: Base de dados Medline, sítios electrónicos de Medicina Baseada na Evidência e publicações da Organização Mundial de Saúde.

Métodos de revisão: Pesquisa de artigos utilizando os termos MeSH albendazole, mebendazole e pyrantel pamoate, publicados entre Janeiro de 2000 e Março de 2011. Foram incluídas citações relacionadas. Foi utilizada a escala de Jadad, para avaliar a robustez metodológica dos ensaios clínicos, e a escala Strength of Recommendation Taxonomy da American Family Physician, para a avaliação da qualidade dos estudos e estabelecer a força de recomendação.

Resultados: Da pesquisa obtiveram-se 746 artigos, 12 preencheram os critérios de inclusão: três revisões sistemáticas, uma meta-análise, quatro ensaios clínicos e quatro normas de orientação clínica. Nas crianças residentes em países com uma prevalência de parasitose intestinal elevada a desparasitação revelou-se benéfica no desenvolvimento estaturo-ponderal. Não se encontrou evidência de benefício em relação ao desenvolvimento cognitivo ou ao aproveitamento escolar. $\mathrm{Na}$ avaliação do impacto sobre a morbilidade os resultados dos diferentes estudos são inconsistentes, no entanto parece haver uma melhoria nos níveis médios de hemoglobina, diminuição da incidência de anemia e de diarreia.

Conclusões: Existe evidência científica de benefício da desparasitação intestinal sistemática, com anti-helmínticos, em crianças residentes em países com prevalência de parasitose intestinal por helmintas transmitidos pelo solo superior a 50\% (SOR B). Em países com baixa prevalência, como Portugal, recomenda-se o tratamento individual baseado no diagnóstico de infecção (SOR C).

Palavras-Chave: Anti-Helmínticos; Enterobius; Ascaris lumbricoides; Trichuris; Ancylostoma; Necator americanus.

\section{INTRODUÇÃO}

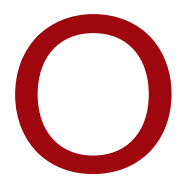

s parasitas gastrointestinais são mais prevalentes em países da África Subsariana, Ásia, América Latina e Caribe, devido ao clima quente e húmido, às más condições higieno-sanitárias (nomeadamente a ausência de rede de fornecimento de água e saneamento básico) e frequente sobrelotação das habitações. ${ }^{1,2} \mathrm{Na}$ América do Norte e Europa estas parasitoses são mais prevalentes em imigrantes e refugiados. ${ }^{1}$

*Médica Interna de Medicina Geral e Familiar do CS Paranhos - UCSP Vale Formoso, ACES Porto Oriental

**Assistente de Medicina Geral e Familiar do CS Paranhos - UCSP Vale Formoso, ACES Porto Oriental
Os vários microrganismos que podem parasitar o tracto gastrointestinal são classificados em dois grandes grupos: os protozoários e os helmintas., ${ }^{1,3}$ No entanto, o alvo da desparasitação intestinal com fármacos são os helmintas. Dentro deste grupo heterogéneo os mais prevalentes são os Enterobius vermicularis (oxiúrios) e os helmintas transmitidos pelo solo (Ascaris lumbricoides, Trichuris trichiuria, Ancylostoma duodenale, Necator americanus). ${ }^{1,4,5}$

Estima-se que 4,5 mil milhões de pessoas estejam em risco de sofrer uma infecção por helmintas transmitidos pelo solo em todo o mundo. ${ }^{4}$ As estimativas apontam para mil milhões de pessoas infectadas por Ascaris lumbricoides, 795 milhões por Trichuris trichiura e 


\begin{tabular}{|c|c|c|c|}
\hline Referências & População & Prevalência da parasitose & $\begin{array}{c}\text { Prevalência da parasitose } \\
\text { por helmintas }\end{array}$ \\
\hline $\begin{array}{l}\text { Gata L. et } a l^{8} \\
\text { (2008) }\end{array}$ & $\begin{array}{r}\mathrm{n}=267 \text { (ambulatório) e } 80 \text { (hospitalar) } \\
\text { Crianças (< } 1 \text { ano) }\end{array}$ & $\begin{array}{r}3,8 \% \text { ambulatório } \\
1,8 \% \text { hospitalar }\end{array}$ & $\begin{array}{l}0 \% \\
0 \%\end{array}$ \\
\hline $\begin{array}{l}\text { Sarmento A. } \\
\text { et } a^{9}(2004)\end{array}$ & $\begin{array}{r}\mathrm{n}=88 \\
\text { Crianças (1-5 anos) seguidas no CS de } \\
\text { Ermesinde }\end{array}$ & $3,4 \%$ & $0 \%$ \\
\hline $\begin{array}{l}\text { Peraboa H. }{ }^{10} \\
(2002)\end{array}$ & $\begin{array}{r}n=216 \\
\text { Crianças (5-14 anos) inscritas em escolas } \\
\text { dos Concelhos de Setúbal e Lisboa }\end{array}$ & $5,1 \%$ & $\begin{array}{r}\text { 3,3\% Trichuris trichiura } \\
\text { 1,9\% Ascaris lumbricoides } \\
1,4 \% \text { Ancylostomidae }\end{array}$ \\
\hline $\begin{array}{l}\text { Cruz A. et al }{ }^{11} \\
(2002)\end{array}$ & $\begin{array}{r}n=471 \\
\text { Crianças (6-11 anos) inscritas em escolas } \\
\text { da cidade do Porto }\end{array}$ & $19,5 \%$ & $\begin{array}{l}5,9 \% \text { Enterobius vermicularis } \\
0,2 \% \text { Ascaris lumbricoides }\end{array}$ \\
\hline
\end{tabular}

Legenda: $\mathrm{n}$ - tamanho da amostra, CS - Centro de Saúde

740 milhões por Ancylostoma duodenale e Necator americanus. ${ }^{6}$

Actualmente, a Organização Mundial de Saúde (OMS) recomenda a desparasitação intestinal sistemática em comunidades com prevalência de infecção intestinal por helmintas transmitidos pelo solo superior a $20 \%$. Esta deve ser realizada uma vez por ano em comunidades com prevalência entre 20 e $50 \%$ (baixo risco) e duas vezes por ano quando a prevalência é superior a $50 \%$ (alto risco). ${ }^{7}$ Os poucos estudos epidemiológicos portugueses encontrados revelam uma taxa de infecção intestinal baixa, com valores de prevalência de parasitose intestinal entre os 3,4\% e os 19\% (Quadro I). ${ }^{8-11}$ A prevalência de parasitose intestinal por helmintas varia entre os $0 \%$ e os 5,9\% (Quadro I). No entanto, este valor pode estar subestimado, uma vez que na maioria dos estudos foi utilizado o exame parasitológico de fezes como meio de diagnóstico e não foi incluído o teste da fita adesiva ou teste de Graham, que é o método considerado de eleição para o diagnóstico da infecção por Enterobius vermiculares. ${ }^{8-11}$

A intensidade da infecção (número de parasitas presentes no tracto gastrointestinal) mede-se através do número de ovos por grama de fezes e está directamente relacionada com a morbilidade destas parasitoses. ${ }^{2}$ As infecções ligeiras são frequentemente assintomáticas enquanto as graves acarretam elevada morbilida- de, com atraso do desenvolvimento estaturo-ponderal e cognitivo, deficiências nutricionais e anemia. ${ }^{4} \mathrm{~A}$ infecção por helmintas transmitidos pelo solo é considerada uma das principais causas mundiais de atraso do desenvolvimento físico e intelectual. ${ }^{2}$

É frequente na prática diária ser-se confrontado com solicitações de prescrição sistemática de desparasitantes intestinais. Da mesma forma, alguns médicos têm a prática da «desparasitação intestinal por rotina». No entanto, as doses e posologias habitualmente utilizadas nesta não são eficazes contra todos os helmintas e os alvos-terapêuticos são sobretudo o Enterobius vermicularis, a Ascaris lumbricoides, o Trichuris trichiuria, o Ancylostoma duodenale e o Necator americanus., ${ }^{3,12,13}$

Desta forma, é fundamental conhecer qual a melhor evidência disponível sobre o benefício desta intervenção. Neste contexto, foi efectuada uma revisão baseada na evidência (RBE), com o objectivo de determinar se a desparasitação intestinal sistemática com anti-helmínticos, em idade pediátrica, influencia o desenvolvimento estaturo-ponderal, cognitivo e o aproveitamento escolar e a morbimortalidade.

\section{MÉTODOS}

Foi efetuada uma pesquisa, nas bases de dados Medline, Guidelines Finder, National Guideline Clearinghouse, Canadian Medical Association Infobase, The Coch- 
rane Library, DARE, Bandolier, TRIP, Índex de Revistas Médicas Portuguesas e publicações da OMS, de revisões sistemáticas, meta-análises, ensaios clínicos controlados e aleatorizados e normas de orientação clínica. Foram também avaliadas, quanto à sua pertinência, as «citações relacionadas» da Pubmed. Utilizaram-se os termos MeSH: albendazole, mebendazole, pyrantel pamoate. Foram seleccionados os artigos publicados entre 1 de Janeiro de 2000 e 31 de Março de 2011, em inglês, espanhol e português. No Índex de Revistas Médicas Portuguesas a pesquisa foi feita com a combinação dos termos da lista dos Descritores em Ciências da Saúde, usando os descritores portugueses: albendazol, mebendazol e pamoato de pirantel.

Foram critérios de inclusão:

- População: Indivíduos com idade inferior a 18 anos de idade.

- Intervenção: Desparasitação intestinal sistemática com fármacos anti-helmínticos.

- Comparação: Fármacos anti-helmínticos versus ausência de intervenção ou placebo.

- Resultados: Desenvolvimento estaturo-ponderal, desenvolvimento cognitivo, aproveitamento escolar e morbimortalidade.

A robustez metodológica dos ensaios clínicos incluídos foi avaliada utilizando a escala de Jadad. ${ }^{14} \mathrm{~A}$ qualidade dos estudos e a força de recomendação foram avaliadas utilizando a escala de Strength of Recommendation Taxonomy (SORT) da American Family Physician. ${ }^{15}$

\section{RESULTADOS}

Da pesquisa obtiveram-se 746 artigos. Destes, 12 preencheram os critérios de inclusão: três revisões sistemáticas, uma meta-análise, quatro ensaios clínicos e quatro normas de orientação clínica. Foram excluídos os artigos repetidos, os não concordantes com os objectivos ou os que não cumpriam os critérios de inclusão. Também foram excluídos os ensaios clínicos controlados e aleatorizados incluídos nas meta-análises e revisões sistemáticas (Figura 1).

De forma a facilitar a interpretação optou-se por analisar os resultados obtidos separadamente para cada um dos objectivos desta RBE: 1) Desenvolvimento estaturo-ponderal;
2) Desenvolvimento cognitivo e aproveitamento escolar e 3) Morbimortalidade. Os artigos encontram-se resumidos em relação às suas principais características nos Quadros II e IV.

\section{1) DESENVOLVIMENTO ESTATURO-PONDERAL}

A meta-análise de Hall et al (2008), concluiu que a desparasitação intestinal sistemática em áreas com uma prevalência de infecção intestinal por nemátodos superior a $50 \%$ originava uma melhoria significativa no ganho de peso e no crescimento. Observou-se um aumento de $0,210 \mathrm{~kg}$ no peso [Intervalo de Confiança (IC) 95\%: 0,17-0,26, $p<0,001]$, um aumento de $0,11 \mathrm{~cm}$ na altura (IC 95\%: 0,03-0,19, $p=0,01$ ) e um aumento de 0,3 $\mathrm{cm}$ na circunferência média do braço (IC 95\%: 0,23$-0,37, p<0,001) .{ }^{16}$ Os estudos incluídos nesta meta-análise apresentam heterogeneidade clínica e estatística,

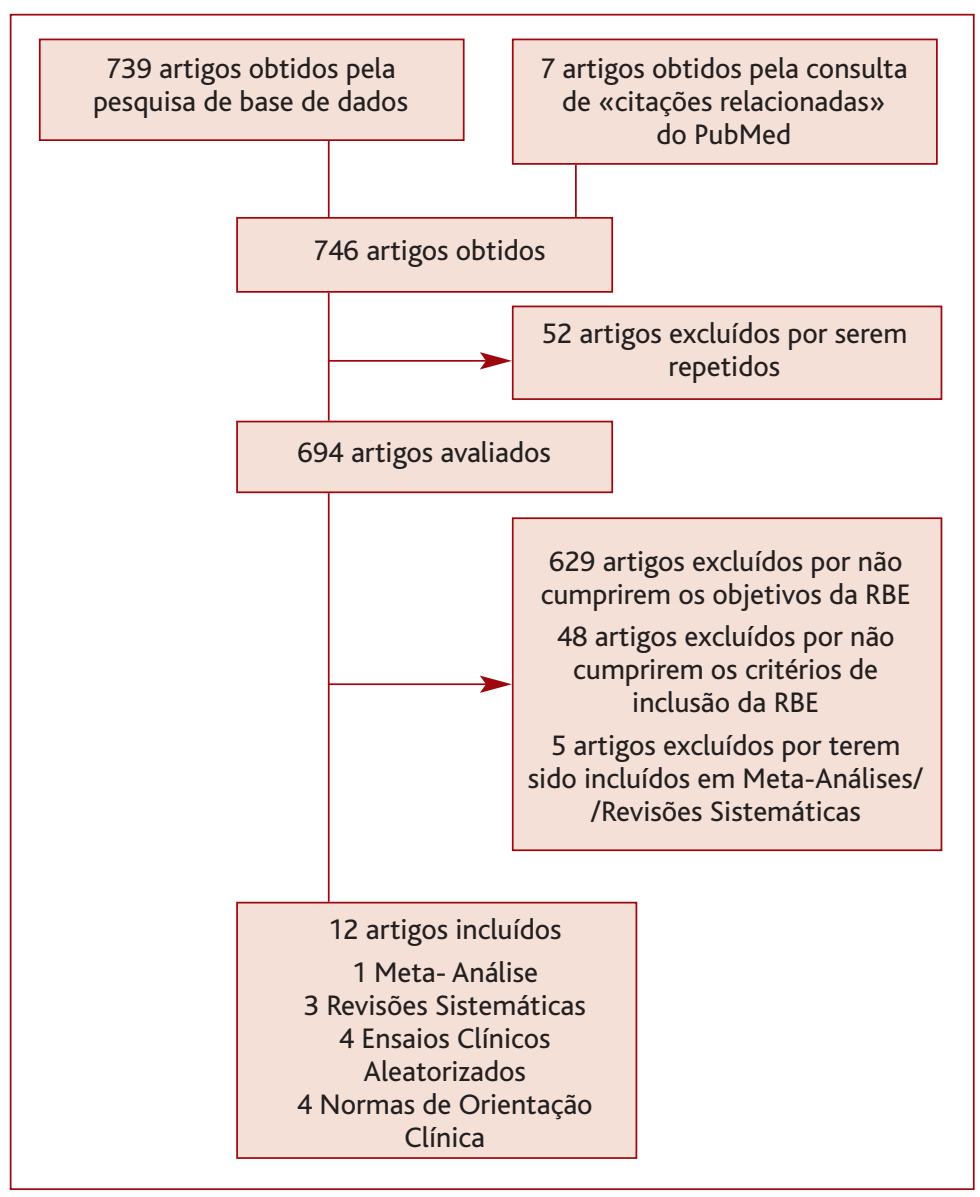

Figura 1. Diagrama da avaliação dos artigos obtidos. 


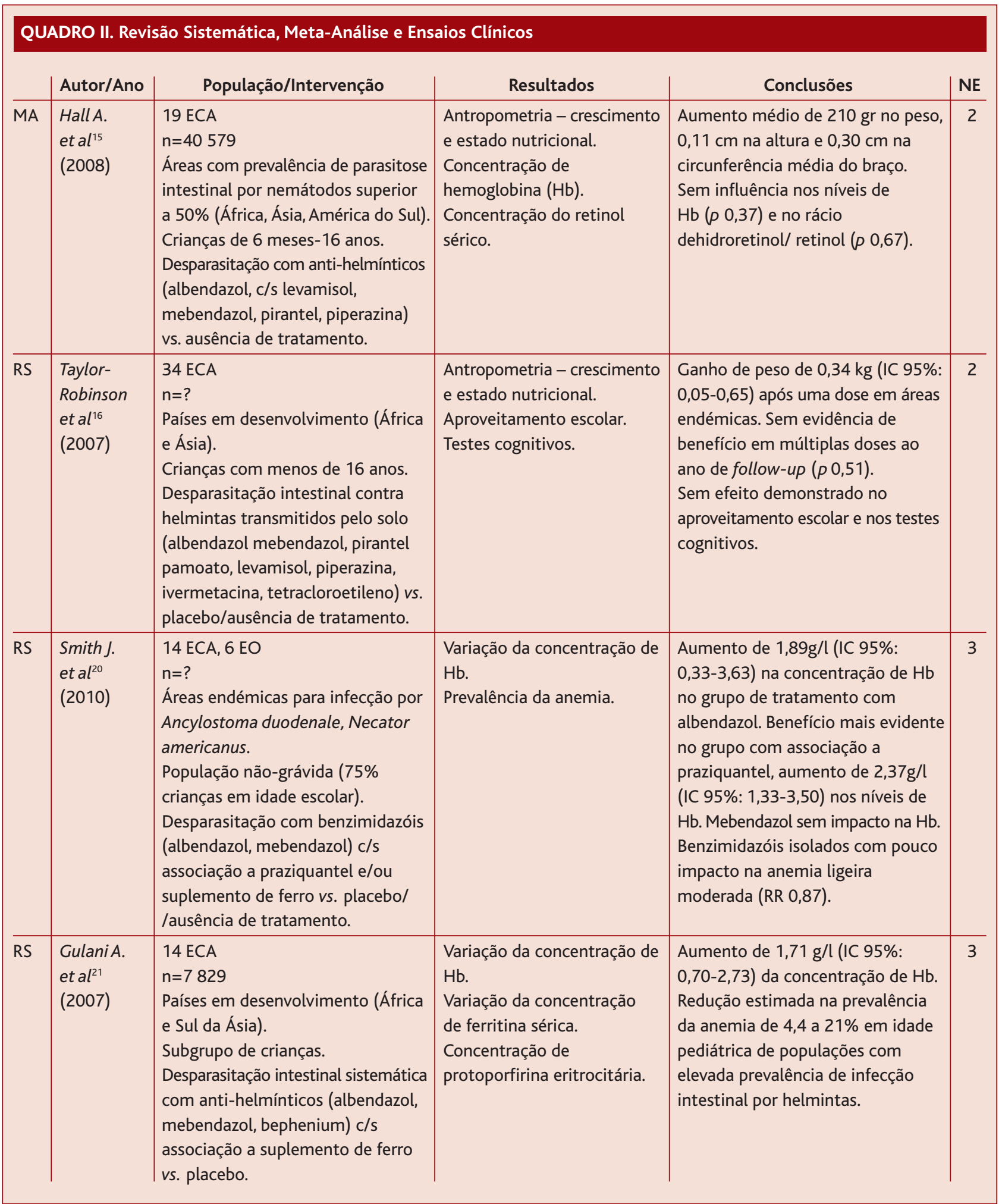


QUADRO II. (Continuação)

\begin{tabular}{|c|c|c|c|c|c|}
\hline & Autor/Ano & População/Intervenção & Resultados & Conclusões & NE \\
\hline ECA & $\begin{array}{l}\text { Elliot } A . \\
\text { et } a l^{19} \\
(2007)\end{array}$ & $\begin{array}{l}\text { ECA } \\
\mathrm{n}=1594 \\
\text { Uganda } \\
\text { Áreas endémicas para infecção por } \\
\text { helmintas. } \\
\text { Crianças na infância precoce } \\
\text { ( } 15 \text { meses). } \\
\text { Albendazol ( } 400 \text { mg de } 3 / 3 \text { meses) } \\
\text { vs. placebo. } \\
\text { Administração concomitante de } \\
\text { múltiplas vacinas (contra } \\
\text { tuberculose, poliomielite, difteria, } \\
\text { tétano, tosse convulsa/pertussis, } \\
\text { Haemophilus influenzae e hepatite } \\
\text { B) e vitamina A (3-3meses). }\end{array}$ & $\begin{array}{l}\text { Incidência de malária, } \\
\text { pneumonia, diarreia, } \\
\text { tuberculose, sarampo, } \\
\text { doenças atópicas. } \\
\text { Concentração sérica de } \\
\text { Hb. } \\
\text { Antropometria. } \\
\text { Desenvolvimento psico-motor. } \\
\text { Desenvolvimento da } \\
\text { linguagem. }\end{array}$ & $\begin{array}{l}\text { Maior incidência de eczema } \\
\text { (RR } 1,3 \text { ). } \\
\text { Sem evidência de diminuição da } \\
\text { incidência de pneumonia, diarreia, } \\
\text { malária e tuberculose. } \\
\text { Sem evidência de benefício no peso, } \\
\text { desenvolvimento psico-motor e da } \\
\text { linguagem. }\end{array}$ & 1 \\
\hline $\mathrm{ECA}$ & $\begin{array}{l}\text { Kirwan P. } \\
\text { et } a^{18} \\
(2010)\end{array}$ & $\begin{array}{l}\text { ECA } \\
\mathrm{n}=320 \\
\text { Nigéria } \\
\text { Áreas endémicas para infecção por } \\
\text { helmintas transmitidos pelo solo e } \\
\text { malária. } \\
\text { Crianças de } 12-59 \text { meses. } \\
\text { Desparasitação intestinal com } \\
\text { albendazol (< } 2 \text { anos de idade - } \\
200 \text { mg, } \geq 2 \text { anos de idade } \\
\text { - } 400 \mathrm{mg} \text { ) de } 4-4 \text { meses associado } \\
\text { a suplemento multivitamínico vs. } \\
\text { placebo (suplemento } \\
\text { multivitamínico). }\end{array}$ & $\begin{array}{l}\text { Infecção por Plasmodium. } \\
\text { Episódios de malária. } \\
\text { Concentração sérica de Hb. } \\
\text { Antropometria. }\end{array}$ & $\begin{array}{l}\text { Menor aumento da prevalência da } \\
\text { infecção por Plasmodium ( } 0,002) \text {. } \\
\text { Sem efeito no número de episódios } \\
\text { de malária. } \\
\text { Aumento dos níveis de } \mathrm{Hb} \text {, sem } \\
\text { significado estatístico ( } 0,445) \text {. } \\
\text { Não se verificou um efeito benéfico } \\
\text { no crescimento (peso e altura). }\end{array}$ & 2 \\
\hline $\mathrm{ECA}$ & $\begin{array}{l}\text { SurD. et al } l^{22} \\
(2004)\end{array}$ & $\begin{array}{l}\text { ECA } \\
\mathrm{n}=702 \\
\text { Favela Indianas. } \\
\text { Áreas endémicas para infecção por } \\
\text { helmintas. } \\
\text { Crianças de } 2-5 \text { anos. } \\
\text { Desparasitação periódica com } \\
\text { albendazol (400 mg de } 6 / 6 \text { meses) } \\
\text { associado a vitamina D vs. placebo } \\
\text { de vitamina D. }\end{array}$ & $\begin{array}{l}\text { Incidência de diarreia } \\
\text { (>3 dejeções/dia ou fezes } \\
\text { aquosas). } \\
\text { Peso. }\end{array}$ & $\begin{array}{l}\text { Redução dos episódios de diarreia } \\
(28 \%) \text {. }\end{array}$ & 2 \\
\hline ECA & $\begin{array}{l}\text { Awasthi S. } \\
\text { et al }{ }^{17} \\
(2008)\end{array}$ & $\begin{array}{l}\text { ECA } \\
\mathrm{n}=3935 \\
\text { Favelas Indianas. } \\
\text { Áreas endémicas para infecção por } \\
\text { helmintas transmitidos pelo solo } \\
\text { Crianças de } 1-5 \text { anos. } \\
\text { Desparasitação intestinal com } \\
\text { albendazol (400mg de } 6 / 6 \text { meses) } \\
\text { associado a vitamina A vs. placebo } \\
\text { (vitamina A). }\end{array}$ & Antropometria. & $\begin{array}{l}\text { Aumento de peso ( } 35 \% \text { ) no grupo } \\
\text { de tratamento, mais evidente nas } \\
\text { crianças desnutridas. } \\
\text { Sem evidência de benefício na } \\
\text { altura. }\end{array}$ & 2 \\
\hline
\end{tabular}

Legenda: RS - revisão sistemática, MA - meta-análise, $\mathrm{n}$ - tamanho da amostra, ECA - estudo controlado e aleatorizado, EO - estudo observacional; Nível de Evidência 2 - estudo de qualidade limitada, evidência orientada para o doente; Nível de Evidência 3 - outra evidência 
pelo que lhe foi atribuído um nível de evidência (NE) 2. A revisão sistemática, realizada por um grupo de trabalho da Cochrane (2007), constatou a existência de uma melhoria significativa no ganho de peso $(0,34 \mathrm{~kg}$, IC 95\%: 0,05-0,64, $p=0,024)$ após uma dose de fármacos anti-helmínticos. No entanto, não houve benefício estatisticamente significativo na altura $(p=0,72)$ e na circunferência média do braço $(p=0,079)$ após uma dose de fármaco. $\mathrm{O}$ mesmo se verificou em relação ao peso $(p=0,51)$ e altura $(p=0,45)$ com a administração de múltiplas doses (Quadro II). ${ }^{17}$ Os estudos incluídos nesta revisão sistemática também apresentam heterogeneidade clínica e estatística, pelo que lhe foi atribuído um NE 2.

O ensaio clínico de Awasthi et al (2008), mostrou um aumento de peso médio de $0,36 \mathrm{~kg}(p=0,0007)$ em crianças indianas tratadas com albendazol, com ganho mais evidente nas crianças desnutridas. Aos dois anos de tratamento verificou-se um aumento no peso de aproximadamente $35 \%$. Não houve benefício estatisticamente significativo na estatura do mesmo grupo $(p=0,11)$ (Quadro II). ${ }^{18}$ Este é um estudo de qualidade limitada por ter sido efectuado sem dupla ocultação (Quadro III), pelo que lhe foi atribuído um NE 2 .

Num ensaio clínico controlado e aleatorizado, realizado por Kirwan et al (2010), em áreas da Nigéria endémicas para malária e com prevalência de infecção por helmintas transmitidos pelo solo superior a $50 \%$, não se encontrou benefício no crescimento (peso e altura) com a administração sistemática de albendazol (Quadro II). ${ }^{19}$ Apesar de ser um estudo de boa qualidade (Quadro III), o período de follow-up foi inadequado pelo que foi classificado com um NE 2.

O ensaio clínico controlado e aleatorizado realizado no Uganda, por Elliot et al (2007), não encontrou evidência de benefício no peso (Quadro II). ${ }^{20}$ Este ensaio clínico é de boa qualidade (Quadro III) pelo que foi classificado com um NE 1.
Em 2006, a OMS publicou um manual intitulado "Preventive chemotherapy in human helminthiasis» (Quadro IV), no qual defende a diminuição da incidência de atraso do crescimento com a desparasitação intestinal para helmintas transmitidos pelo solo. ${ }^{7}$ Este manual é baseado na opinião de peritos pelo que tem um NE 3.

\section{2) DESENVOLVIMENTO COGNITIVO E APROVEITAMENTO ESCOLAR}

Na revisão sistemática da Cochrane (2007) não foi demonstrado benefício no aproveitamento escolar nem nos testes cognitivos (NE 2). ${ }^{17} \mathrm{O}$ ensaio clínico controlado e aleatorizado de Elliot et al (2007) também não mostrou benefício da desparasitação intestinal sistemática no desenvolvimento psico-motor ou da linguagem (NE 1) ${ }^{20} \mathrm{Na}$ sua publicação «Preventive chemotherapy in human helminthiasis» (2006), a OMS defende que a desparasitação intestinal dirigida a helmintas transmitidos pelo solo melhora a capacidade cognitiva e diminui o absentismo escolar e laboral (NE 3). ${ }^{7}$

\section{3) MORBIMORTALIDADE}

A OMS defende que a desparasitação intestinal dirigida a helmintas transmitidos pelo solo diminui a morbilidade, a fadiga crónica e o absentismo escolar e laboral (NE 3). ${ }^{7}$ Em relação ao benefício da desparasitação intestinal sistemática na diminuição de doenças sistémicas, o ensaio realizado na Nigéria, por Kirwan et al, verificou que o albendazol está associado a um menor aumento da prevalência da infecção por Plasmodium

\begin{tabular}{|l|l|c|c|c|c|}
\hline \multicolumn{2}{|l|}{ QUADRO III. Robustez metodológica dos ensaios clínicos aleatorizados incluídos segundo } \\
a escala de Jadad ${ }^{14}$
\end{tabular}




\begin{tabular}{|c|c|c|}
\hline Referência & Recomendações & $\begin{array}{l}\text { Força de } \\
\text { recomendação }\end{array}$ \\
\hline $\begin{array}{l}\text { MeReC - NPC/NICE. } \\
\text { Actuação na infecção por } \\
\text { Enterobius vermicularis em } \\
\text { Cuidados de Saúde Primários }{ }^{24} \\
\text { (2008). }\end{array}$ & $\begin{array}{l}\text { O tratamento com anti-helmínticos só está recomendado x confirmação } \\
\text { diagnóstica (visualização do verme ou detecção de ovos). } \\
\text { Se o tratamento estiver indicado o mebendazol é o fármaco de eleição e toda } \\
\text { a família deve ser tratada. }\end{array}$ & $C$ \\
\hline $\begin{array}{l}\text { NHS - CKS. } \\
\text { Clinical Topic - Oxiúrios } \\
\text { (Enterobius vermicularis) } \\
\text { (2007). }\end{array}$ & $\begin{array}{l}\text { O tratamento com anti-helmínticos só está recomendado após confirmação } \\
\text { diagnóstica (visualização do verme ou detecção dos ovos). }\end{array}$ & C \\
\hline $\begin{array}{l}\text { NHS - CKS. } \\
\text { Clinical Topic - Lombrigas } \\
\text { (Ascaris lumbricoides) } \\
\text { (2007). }\end{array}$ & $\begin{array}{l}\text { Deve ser obtida uma amostra de fezes, em pessoas que apresentam sintomas } \\
\text { gastrointestinais inespecíficos persistentes por mais } 10-14 \text { dias (tais como } \\
\text { vómitos, diarreia, cólicas) e que estiveram em áreas endémicas nos últimos } \\
\text { dois anos, para confirmar ou excluir o diagnóstico de Ascaris lumbricoides } \\
\text { antes de se iniciar o tratamento. }\end{array}$ & $C$ \\
\hline $\begin{array}{l}\text { OMS. } \\
\text { Quimioterapia Preventiva nas } \\
\text { Infeções por Helmintas nos } \\
\text { Humanos } \\
\text { (2006). }\end{array}$ & $\begin{array}{l}\text { Desparasitação intestinal dirigida a helmintas transmitidos pelo solo leva a } \\
\text { uma diminuição da morbilidade (anemia por défice de ferro, fadiga crónica, } \\
\text { obstrução intestinal, atraso do crescimento, diminuição da capacidade } \\
\text { cognitiva), diminuição do absentismo escolar e laboral e diminuição da } \\
\text { mortalidade. } \\
\text { Quando a prevalência de infecção em crianças em idade escolar excede os } \\
20 \% \text { está recomendado a quimioterapia preventiva e sistemática das crianças } \\
\text { em idade pré-escolar e escolar, grávidas, puérperas ou mulheres a amamentar } \\
\text { e em adultos com ocupação de risco. } \\
\text { Em áreas de prevalência inferior a } 20 \% \text { não está recomendado a quimioterapia } \\
\text { preventiva de larga escala e sistemática. Nestes casos o tratamento deve ser } \\
\text { selectivo (após diagnóstico). }\end{array}$ & C \\
\hline
\end{tabular}

Legenda: MeReC - publicação do National Prescribing Centre (NPC) em colaboração com NICE (National Institute for Health and Clinical Excellence), NHS - CKS - National Health Service - Clinical Knowledge Summaries.

que o grupo de placebo $(p=0,002)$ mas não houve evidência de benefício no número de episódios de malária $(p=1)$ (Quadro II) (NE 2). ${ }^{19} \mathrm{O}$ ensaio clínico controlado e aleatorizado de Elliot et al (2007) verificou, no ramo do estudo sujeito a intervenção, uma maior incidência de eczema, sem evidência de diminuição da incidência de pneumonia, malária ou tuberculose (NE 1). ${ }^{20}$

A meta-análise de Hall et al (2008) não mostrou melhoria significativa dos níveis de hemoglobina $(\mathrm{Hb})$ $(p=0,37)(\mathrm{NE} 2)^{16}$ e a revisão sistemática, realizada por Smith et al, objectivou um aumento de concentração média de $\mathrm{Hb}$ no ramo do estudo sujeito a tratamento. ${ }^{21}$
Este aumento foi de 1,89g/L (IC 95\%: 0,33-3,63) no grupo de tratamento com albendazol e de 2,37g/L (IC 95\%: 1,33-3,50) no grupo de tratamento com albendazol e praziquantel. ${ }^{21}$ No entanto, os benzimidazóis isolados revelaram impacto diminuto na anemia moderada (Risco Relativo (RR 0,87), sendo maior na associação com praziquantel (RR 0,61). ${ }^{21}$ Esta revisão tem um NE 3 por estar orientada para a doença. A revisão sistemática de Gulani et al (2007) evidenciou um aumento de 1,71 g/L (IC 95\%: 0,70-2,73) na concentração de Hb, estimando uma redução na prevalência da anemia em idade pediátrica de populações com elevada prevalência de in- 
fecção intestinal por helmintas de 4,4\% a 21\% (Quadro II). ${ }^{22}$ Este estudo tem um NE 3 por estar orientado para a doença. O ensaio clínico controlado e aleatorizado realizado na Nigéria e publicado em 2010 verificou um aumento dos níveis de $\mathrm{Hb}$ sem significado estatístico $(p=0,445)(\mathrm{NE} 2) .{ }^{19}$

Relativamente à diarreia, os resultados encontrados são inconsistentes. O ensaio clínico controlado e aleatorizado realizado no Uganda, por Elliot et al (2007), concluiu não existir evidência de diminuição da incidência de diarreia (NE 1), enquanto o ensaio clínico controlado e aleatorizado realizado em favelas indianas e publicado em 2004 verificou uma redução dos episódios de diarreia em $28 \%$ com o albendazol. ${ }^{20,23}$ Este último tem um NE 2 porque apesar de ser um estudo de boa qualidade o período de follow-up foi inadequado (Quadro III).

É difícil de quantificar a mortalidade atribuída à infecção intestinal por nemátodos devido à clínica inespecífica destas infecções e à escassez de dados epidemiológicos por carência de redes de notificação nos países em desenvolvimento. ${ }^{24}$ Assim, não foram encontrados artigos que estimassem os efeitos da desparasitação na mortalidade. No entanto, a OMS defende que a desparasitação intestinal sistemática em países com elevada prevalência diminui a mortalidade (NE 3). ${ }^{7}$

\section{CONCLUSÕES}

A maioria dos artigos incluídos nesta revisão teve como base populações pediátricas de países em desenvolvimento com prevalência de parasitose intestinal superior a $50 \%$. Clinicamente são heterogéneos por uso de anti-helmínticos e esquemas posológicos diferentes. Alguns estudos administraram, concomitantemente, outros compostos (por exemplo, vitamina D, vacinas ou ferro) que podem confundir os resultados. Exceptuando-se o benefício no peso, os restantes resultados neles obtidos são inconsistentes.

Há evidência de benefício da desparasitação intestinal sistemática em crianças residentes em países com prevalência de parasitose intestinal por helmintas transmitidos pelo solo superior a $50 \%$ (SOR B). Não foram encontrados estudos que avaliassem o benefício da desparasitação sistemática em crianças saudáveis, assintomáticas e residentes em países de baixa prevalência, como parece ser o caso de Portugal. Nestes, o tra- tamento deve ser selectivo e baseado no diagnóstico de infecção (SOR C) (Quadro IV).7, 24-26

\section{REFERÊNCIAS BIBLIOGRÁFICAS}

1. Harhay MO, Horton J, Olliaro PL. Epidemiology and control of human gastrointestinal parasites in children. Expert Rev Anti Infect Ther 2010 Feb; 8 (2): 219-34.

2. Bethony J, Brooker S, Albonico M, Geiger SM, Loukas A, Diemert D, et al. Soil-transmitted helminth infections: ascariasis, trichuriasis, and hookworm. Lancet 2006 May 6; 367 (9521): 1521-32.

3. Fernandes S, Beorlegui M, Brito MJ, Rocha G; Sociedade de Infeciologia Pediátrica. Linhas de Orientação Sobre Parasitoses Intestinais. Sociedade Portuguesa de Pediatria. 2011. Disponível em: http://www. spp.pt/UserFiles/file/Seccao_Infecciologia/PARASITOSES_INTESTINAIS_Protocolo.pdf [acedido em 16/06/2011].

4. Keiser J, Utzinger J. Efficacy of current drugs against soil-transmitted helminth infections: systematic review and meta-analysis. JAMA 2008 Apr 23; 299 (16): 1937-48.

5. Kucik CJ, Martin GL, Sortor BV. Common Intestinal Parasites. Am Fam Physician 2004 Mar; 69 (5): 1161-8.

6. World Health Organization. Intestinal Worms: soil transmitted helminths. Disponível em: http://www.who.int/intestinal_worms/en/ [acedido em 16/06/2011].

7. World Health Organization. Preventive chemotherapy in human helminthiasis: Coordinated use of anthelminthic drugs in control interventions: a manual for health professionals and programme managers. France:WHO; 2006. Disponível em: http://whqlibdoc.who.int/publications/2006/9241547103_eng.pdf [acedido em 02/04/2011].

8. Gata L, Gomes L, Pereira MH, Tomé R, Salgado M. Parasitoses intestinais em crianças e adultos: Estudos realizados em laboratórios do ambulatório e hospitalar. Rev Saúde Infant 2008 Dez; 30 (3): 106-9.

9. Sarmento A, Costa JM, Valente CAP, Teixeira ME. Infecção por parasitas intestinais numa população pediátrica. Acta Pediatr Port 2004; 35 (4): 307-11.

10. Peraboa HG. Helmintas intestinais em alunos de escolas primárias nos concelhos de Lisboa e Setúbal [dissertação de mestrado]. Lisboa: Universidade Nova de Lisboa; 2002.

11. Cruz A, Cabral M, Sousa MI, Azeredo Z. Parasitoses intestinais - estudo transversal em crianças de escolas do $1^{\circ}$ ciclo da cidade do Porto. Arq Med 2002; 16: 211-8.

12. Infarmed. Prontuário Terapêutico. Lisboa: Infarmed; 2010. Medicamentos Anti - infecciosos; p 21-72.

13. Varandas L. Parasitoses: abordagem global. In: Amaral J. Tratado de Clínica Pediátrica. Amadora: Abbott Laboratórios; 2008. p.1506-17.

14. Jadad AR, Moore RA, Carroll D, Jenkinson C, Reynolds DJ, Gavaghan DJ, et al. Assessing the quality of reports of randomized clinical trials: is blinding necessary? Control Clin Trials 1996 Feb; 17 (1): 1-12.

15. Ebell MH, Siwek J, Weiss BD, Woolf SH, Susman J, Ewingman B, et al. Strength of Recommendation Taxonomy (SORT): a patient-centered approach to grading evidence in the medical literature. Am Fam Physician 2004 Feb 1; 69 (3): 548-56.

16. Hall A. Hewitt G. Tuffrey $V$, de Silva N. A review and meta-analysis of the impact of intestinal worms on child growth and nutrition. Matern Child Nutr 2008 Apr; 4 Suppl 1: S118-236. 
17. Taylor-Robinson DC, Jones AP, Garner P. Deworming drugs for treating soil-transmitted intestinal worms in children: effects on growth and school performance. Cochrane Database of Syst Rev 2007 Oct 17; (4): CD000371.

18. Awasthi S, Peto R, Pande VK, Fletcher RH, Read S, Bundy DA. Effects of deworming on malnourished preschool children in India: an open-labelled, cluster-randomized trial. PLoS Negl Trop Dis 2008 Apr 16; 2 (4): e223.

19. Kirwan P, Jackson AL, Asaolu SO, Molly SF, Abiona TC, Bruce MC, et al. Impact of repeated four-monthly anthelmintic treatment on Plasmodium infection in preschool children: a double-blind placebo-controlled randomized trial. BMC Infect Dis 2010 Sep 21; 10: 277.

20. Elliott AM, Kizza M, Quigley MA, Ndibazza J, Nampijja M, Muhangi L, et al. The impact of helminths on the response to immunization and on the incidence of infection and disease in childhood in Uganda: design of a randomized, double-blind, placebo-controlled, factorial trial of deworming interventions delivered in pregnancy and early childhood. Clin Trials 2007; 4 (1): 42-57.

21. Smith JL, Brooker S. Impact of hookworm infection and deworming on anemia in non-pregnant populations: a systematic review. Trop Med Int Health 2010 Jul; 15 (7): 776-95.

22. Gulani A, Nagpal J, Osmond C, Sachdev HP. Effect of administration of intestinal anthelmintic drugs on haemoglobin: systematic review of randomised controlled trials. BMJ 2007 May 26; 334 (7603): 1095.

23. Sur D, Saha DR, Manna B, Rajendran K, Bhattacharya SK. Periodic deworming with albendazole and its impact on growth status and diarr- hoeal incidence among children in an urban slum of India. Trans R Soc Trop Med Hyg 2005 Apr; 99 (4): 261-7.

24. Brooker S. Estimating the global distribution and disease burden of intestinal nematode infections: adding up the numbers - A review. Int J Parasitol 2010 Aug 15; 40 (10): 1137-44.

25. National Prescribing Center. Management of threadworms in primary care. MeReC Bull 2008; 18 (4): 11-3.

26. National Health Service. Threadworm. Clinical Knowledge Summaries [internet]. 2007. Disponível em: http://www.cks.nhs.uk/threadworm\# [acedido em 02/04/20111].

27. National Health Service. Roundworm. Clinical Knowledge Summaries [internet]. 2007. Disponível em: http://www.cks.nhs.uk/roundworm\# [acedido em 02/04/2011].

\section{CONFLITOS DE INTERESSE}

As autoras declaram ausência de conflitos de interesses e financiamento do estudo.

\section{ENDEREÇO PARA CORRESPONDÊNCIA}

Vanessa Xavier

Rua de Vale Formoso, N. ${ }^{\circ} 466$

4200-510 Paranhos Porto

E-mail: vanessaxavier.mgf@gmail.com

Recebido em 27/06/2011

Aceite para publicação em 23/02/2012

\section{ABSTRACT}

\section{SYSTEMATIC INTESTINAL DEWORMING IN PEDIATRIC POPULATIONS: AN EVIDENCE BASED REVIEW}

Objectives: To determine whether systematic intestinal deworming with anthelmintic medications in pediatric populations affects height, weight, cognitive and academic performance, morbidity and mortality in children.

Data Sources: MEDLINE database, electronic evidence-based medicine websites and World Health Organization publications. Review Methods: A search for articles using the MeSH terms albendazole, mebendazole, and pyrantel pamoate, published between January, 2000 and March, 2011 was conducted. Some related citations were also included. The Jadad Score was used to assess the quality of randomized clinical trials. The Strength of Recommendation Taxonomy (SORT) scale of the American Family Physician was used to assess the corresponding quality and strength of recommendations.

Results: The search produced a total of 746 articles, of which 12 met the inclusion criteria. These included three systematic reviews, one meta-analysis, four clinical trials and four clinical practice guidelines. In children living in countries with a high prevalence of intestinal parasite infection, deworming proved to be beneficial in promoting height and weight gain. With reference to cognitive development and school performance, no evidence was found as to the benefits of deworming. Studies show inconsistent results in terms of morbidity. However, there seems to be an improvement in median hemoglobin levels, as well as a decrease in anemia and diarrhea.

Conclusions: There is evidence for the benefits of systematic intestinal deworming with anthelmintics in children living in countries with a prevalence of intestinal parasite infection higher than $50 \%$ resulting from soil-transmitted helminths (SOR B). In countries with a low prevalence of infection, such as Portugal, individual treatment based on a diagnosis of infection is recommended (SOR C).

Keywords: Anthelmintics; Enterobius; Ascaris lumbricoides; Trichuris; Ancylostoma; Necator americanus. 